\title{
EFEITOS DOS MÉTODOS DE ABATE DE BOVINOS NA EFICIÊNCIA DA SANGRIA ${ }^{1}$
}

\author{
Roberto de Oliveira ROÇA ${ }^{2, *}$, Carlos Roberto PADOVANI ${ }^{3}$, Mário Carlos de FILIPI ${ }^{2}$, Erich $\mathrm{SCHWACH}^{2}$, \\ Alberto UEMI ${ }^{2}$, Roberto Takeo SHINKAI ${ }^{2}$, Germano Francisco BIONDI ${ }^{4}$
}

\begin{abstract}
RESUMO
O objetivo do presente trabalho foi avaliar os efeitos dos métodos de abate de bovinos com insensibilização prévia através de marreta e pistola pneumática de penetração e sem insensibilização, realizada pelo método kasher, na eficiência da sangria. A eficiência da sangria foi avaliada pela determinação da hemoglobina no sangue e na carne. Foram utilizados 240 bovinos sãos, da raça Nelore, abatidos em matadouro-frigorífico sob Serviço de Inspeção Federal, sendo 60 animais abatidos pelo método judaico (kasher), 60 animais insensibilizados através de marreta, 60 animais insensibilizados através de pistola pneumática de penetração e 60 animais insensibilizados através de pistola pneumática de penetração seguida por estimulação elétrica. Foram colhidas amostras de sangue após a sangria dos animais e de carne, na porção torácica do músculo Longus colli, imediatamente após a chegada da carcaça à câmara frigorífica. Os animais abatidos pelo método kasher apresentaram menor teor de hemoglobina residual na carne e melhor eficiência da sangria. A utilização da estimulação elétrica imediatamente após a sangria dos animais atordoados por pistola pneumática, afetou negativamente a eficiência da sangria.
\end{abstract}

Palavras-chave: abate de bovinos; abate kasher; eficiência da sangria.

\section{SUMMARY}

EFFECTS OF SLAUGHTER METHODS ON EFFICIENCY. The objective of this work was to evaluate the bovine slaughter methods, with previous stunning through stone-hammer, pneumatic powered stunners and no stunning, carried out the kasher method on bleeding efficiency. Bleeding efficiency was evaluated through muscle haemoglobin/blood haemoglobin ratio using blood taken at bleeding and muscle samples taken before chilling. Two hundred and forty Nelore steers were slaughtered in a commercial slaughterhouse under Federal Inspection Service, where 60 animals were slaughtered by the Jewish method (kasher), 60 animals were stunned through stone-hammer, 60 animals were stunned through the pneumatic powered stunners and 60 animals were stunned through pneumatic powered stunners followed by electrical stimulation. The animals slaughtered by the kashermethod showed less residual haemoglobin retained in the muscle and better bleeding efficiency. Electrical stimulation utilization after animal bleeding were stunned by the pneumatic powered stunners had a negative effect on bleeding efficiency.

Keywords: slaughter; kasher; bleeding efficiency.

\footnotetext{
${ }^{1}$ Recebido para publicação em 30/04/01. Aceito para publicação em 23/07/01.

${ }^{2}$ Laboratório de Tecnologia dos Produtos de Origem Animal. FCA. UNESP, Campus de Botucatu. E-mail:robertoroca@fca.unesp.br - Caixa Postal, 237, CEP 18603-970 - Botucatu - SP.

${ }^{3}$ Departamento de Bioestatística. IB-UNESP, Campus de Botucatu. ${ }^{4}$ Departamento de Higiene Veterinária e Saúde Pública - FMVZ - UNESP, Campus de Botucatu.

${ }^{*}$ A quem a correspondência deve ser enviada.
}

\section{1- INTRODUÇÃO}

A eficiência da sangria pode ser definida como o volume de sangue residual ou retido nos músculos após o abate. Considerando uma variação individual muito acentuada no teor de hemoglobina sangüínea, a relação entre a hemoglobina sangüínea e a hemoglobina residual no músculo é uma forma segura de avaliação da eficiência da sangria, cujos resultados são expressos em $\mathrm{mL}$ de sangue retido no músculo por $100 \mathrm{~g}$ de músculo [26].

O volume de sangue de bovinos é estimado em 6,4 a 8,2 litros $/ 100 \mathrm{~kg}$ de peso vivo [3, 16]. A quantidade de sangue obtida na sangria com o animal deitado é aproximadamente de 3,96 litros $/ 100 \mathrm{~kg}$ de peso vivo e com a utilização do trilho aéreo é de 4,42 litros/100kg de peso vivo [3]. Numa boa sangria, necessária para a obtenção de uma carne com adequada capacidade de conservação, é removido cerca de $60 \%$ do volume total de sangue, sendo que o restante fica retido nos músculos $(10 \%)$ e vísceras $(20-25 \%)[14,22,32]$.

O sangue tem $\mathrm{pH}$ alto $(7,35-7,45)[16]$ e, devido ao grande teor protéico, tem uma rápida putrefação [19]. Logo, a capacidade de conservação da carne mal sangrada é muito limitada. Além disso, constitui um problema de aspecto para o consumidor [3, 14]. Portanto, a eficiência da sangria pode ser considerada uma exigência importante das operações de abate para obtenção de um produto de alta qualidade [37].

Vários fatores são responsáveis pela eficiência da sangria. Podem ser citados o estado físico do animal antes do abate, método de atordoamento e o intervalo entre o atordoamento e a sangria. Todas as enfermidades que debilitam o sistema circulatório afetam a sangria. As enfermidades febris, agudas, provocam vasodilatação generalizada o que impede uma sangria eficiente. O mesmo é observado em animais abatidos em estado agônico, tendo em vista que o sistema circulatório está notadamente alterado [3, 20].

O banho de aspersão tem sido apontado como um procedimento capaz de melhorar a sangria através da vasoconstricção periférica que ela possa provocar [2], porém de acordo com ROÇA \& SERRANO [27], esta etapa do abate de bovinos não afeta a eficiência da sangria ou o teor de hemoglobina retido nos músculos.

Com relação aos efeitos dos métodos de insensibilização na eficiência da sangria, os trabalhos científicos são escassos. O atordoamento do animal, por qualquer método, produz uma elevação da pressão sangüínea no sistema arterial, venoso e capilares, e dá um aumento transitório nos batimentos cardíacos [34], fato- 
res que favorecem a sangria. $O$ volume de sangue coIhido também é maior se a sangria é realizada imediatamente após a insensibilização. A esse respeito, VIMINI et al $[35,36]$ estabeleceram que o volume de sangue colhido é inversamente proporcional ao intervalo entre $\mathrm{o}$ atordoamento e a sangria.

A importância da sangria imediata é evidente quando se verifica que a velocidade de um fluxo de um vaso cortado é 5 a 10 vezes mais rápido do que no vaso íntegro e somente depois de perder-se muito sangue é que a pressão sangüínea começa a cair [34]. Na Argentina, o intervalo máximo permitido é de dois minutos para bovinos [1] e na Holanda, 30 segundos após eletronarcose em ovinos [17]. No Brasil, o Serviço de Inspeção Federal recomenda um intervalo máximo de 1 minuto para bovinos [5].

Um problema relacionado com a sangria é o aparecimento de hemorragias musculares caracterizadas por petéquias, listras ou equimoses em várias partes da musculatura, provocada por aumento da pressão sangüínea e ruptura capilar [17, 34]. Vários fatores são responsáveis por estas alterações, como o aumento do intervalo entre o atordoamento e a sangria [34], o estado de tensão dos animais no momento do abate [12], traumatismos, infecções e ingestão de substâncias tóxicas [33].

O objetivo do presente trabalho foi avaliar os efeitos dos métodos de abate de bovinos com insensibilização prévia através de marreta e pistola pneumática de penetração e sem insensibilização, realizada pelo método kasher. O termo kosher ou kasher significa preparado de acordo com as leis judaicas de alimentação [23, 24, 25]. O abate kasher ou schechita envolve a contenção do animal, estiramento da cabeça através de um ganho, e uma incisão, sem movimentos bruscos, entre a cartilagem cricóide e a laringe [21], cortando a pele, músculos, traquéia, esôfago, veias jugulares e artérias carótidas [24] e às vezes chegando próximo às vértebras cervicais [28]. Esta operação tem como objetivo, segundo REGENSTEIN \& REGENSTEIN [24], permitir a máxima remoção de sangue.

\section{2 - MATERIAL E MÉTODOS}

\section{1 - Material}

Foram utilizados 240 bovinos sãos, da raça Nelore sendo divididos em quatro grupos de 60 animais, de acordo com o método de abate:

- Grupo I: animais abatidos pelo método judaico (kasher)

- Grupo II: animais insensibilizados com marreta;

- Grupo III: animais insensibilizados através de pistola pneumática de penetração

- Grupo IV: animais insensibilizados através de pistola pneumática de penetração seguida por estimulação elétrica.

Os animais foram abatidos em matadouro-frigorífi- co sob Serviço de Inspeção Federal, em Bauru, S.P. (Grupos I, II e III), e em Lins, S.P. (Grupo IV). Cada grupo de animais foi composto de 6 lotes, contendo 9 a 11 animais por lote.

Os animais foram transportados por via rodoviária e após serem submetidos à inspeção ante-mortem e dieta hídrica por 18 a 30 horas, foram abatidos, suspensos através de guincho elétrico e processados com o auxílio de transportador aéreo automático. O fluxograma geral do abate está sintetizado na Figura 1.

O banho de aspersão dos animais foi realizado com água clorada à temperatura ambiente durante 6 a 10 minutos. $\mathrm{O}$ abate dos 60 animais pelo métodos kasher foi realizado por magareferes israelenses, treinados e autorizados para essa finalidade. $O$ animal foi contido na área de vômito para a realização da degola.

Nos 180 animais restantes, a insensibilização com marreta (60 animais) ou pistola pneumática (120 animais; Grupos III e IV) foi realizada no boxe de atordoamento.

A pistola pneumática possuía as seguintes características: velocidade do dardo: $44,3 \mathrm{~m} / \mathrm{s}$; energia de impacto: 294,4 joules, pressão de trabalho: $12 \mathrm{~kg} / \mathrm{cm}^{2}$, curso total do dardo: $80 \mathrm{~mm}$; saída do dardo: $50 \mathrm{~mm}$; diâmetro do dardo: $12 \mathrm{~mm}$.

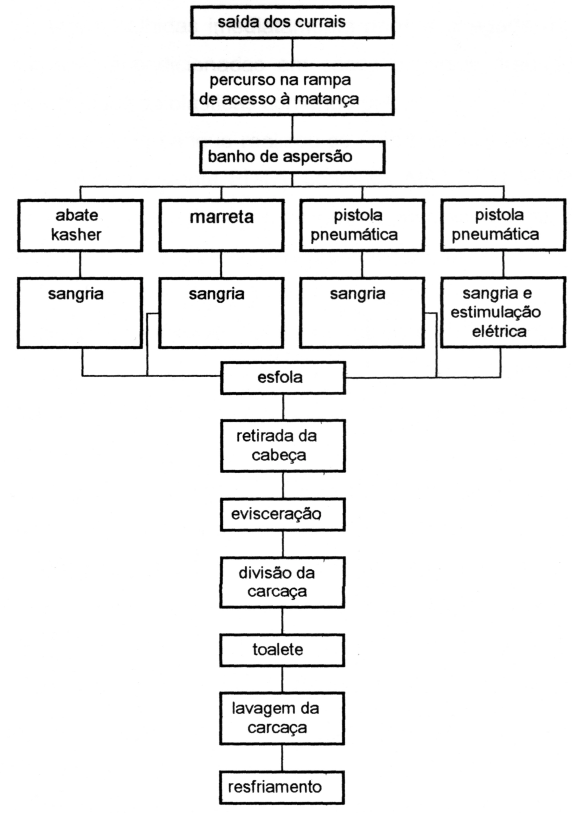

FIGURA 1. Fluxograma geral do abate

Após o atordoamento, os animais foram suspensos através de uma das patas e a sangria realizada com duas facas previamente esterilizadas em água à temperatura de ebulição.

Em 60 animais abatidos com pistola pneumática foi realizada a estimulação elétrica (Grupo IV), por 1 minuto e 22 segundos, com corrente elétrica de $80 \mathrm{~V}$, $200 \mathrm{~mA}, 120 \mathrm{pps}, 60 \mathrm{~Hz}$, imediatamente após o corte dos vasos, na canaleta de sangria. 
A lavagem das carcaças foi realizada por 3 operadores, sendo 2 no piso e 1 em plataforma elevada, com mangueiras de alta pressão. A velocidade média de abate foi de 120 animais por hora no abate com insensibilização e em torno de 80 bovinos por hora no método kasher.

\section{2 - Métodos}

\subsection{1 - Amostragem e avaliação da eficiência da sangria}

As amostras, em torno de $500 \mathrm{~g}$ da porção torácica do músculo Longus colli, foram colhidas imediatamente após a chegada da carcaça à câmara frigorífica, acondicionadas em sacos plásticos, transportadas em caixa térmica com bolsas plásticas contendo gelo.

A colheita do sangue realizou-se com o auxílio de frascos de $10 \mathrm{~mL}$ contendo o anticoagulante EDTA, conforme MATOS \& MATOS [18]; colhendo-se aproximadamente $5 \mathrm{~mL}$ de sangue de cada animal, 5 segundos após o corte dos vasos sangüíneos, na área de sangria.

As avaliações efetuadas foram as seguintes:

- Hemoglobina sangüínea: avaliada através da determinação da cianometahemoglobina sangüínea, de acordo com o método básico de DRABKIN \& AUSTIN [7], com as modificações citadas por DACIE \& LEWIS e MATOS \& MATOS $[6,18]$.

- Hemoglobina no músculo: a determinação da cianometahemoglobina no músculo foi realizada através da extração de mioglobina e hemoglobina com solução fisiológica tamponada $(\mathrm{pH}=7,4)$; a separação das duas proteínas foi conduzida conforme KARASZ et al [15], por precipitação da hemoglobina com sulfato de amônio e a leitura espectrofotométrica realizada a 422nm [27], empregando-se como amostra a solução de hemoglobina mais mioglobina e como "branco" a solução de mioglobina.

- Mioglobina: a determinação da cianometamioglobina no músculo foi realizada através da extração de mioglobina e hemoglobina com solução fisiológica tamponada ( $\mathrm{pH}=7,4)$; a separação das duas proteínas foi conduzida conforme KARASZ et al [15], por precipitação da hemoglobina com sulfato de amônio e com leitura espectrofotométrica a 422nm, empregando-se como amostra a solução de mioglobina e como "branco" a solução de reagente de coloração mioglobina.

- Pigmentos totais: realizada da mesma forma que a avaliação da hemoglobina, sendo utilizado como branco a solução contendo apenas os reagentes de cor.

- Eficiência da sangria: o cálculo da eficiência da sangria foi determinado pela equação: $\mathrm{mL}$ de sangue $/ 100 \mathrm{~g}$ de músculo $=(\mathrm{g} / 100 \mathrm{~g}$ de hemoglobina no músculo: $\mathrm{g} / \mathrm{dL}$ de hemoglobina no sangue) $\times 100$, conforme ROÇA \& SERRANO [27].

\subsection{2 - Avaliação estatística}

O delineamento experimental adotado para as avaliações da eficiência da sangria foi o de blocos inteiramente casualisados. As médias dos quatro grupos de 60 animais cada um, foram comparadas através do Teste de Tukey, conforme SNEDECOR \& COCHRAN e SAS [29, 31].

\section{3 - RESULTADOS E DISCUSSÃO}

Os resultados obtidos na avaliação do processo de sangria de bovinos abatidos estão apresentados na Tabela 1 , e a correlação entre os parâmetros analisados, na Tabela 2.

O processo de sangria produz uma hipovolemia e desta forma é instalado o choque hemorrágico [10, 13, 30]. Os eventos hemodinâmicos do choque são: diminuição do volume sangüíneo, decréscimo do retorno ao coração, diminuição do rendimento cardíaco, queda da pressão arterial, vasoconstricção intensa e persistente, hipóxia tecidual isquêmica, aumento da permeabilidade capilar, passagem de plasma para o interstício e decréscimo do retorno sangüíneo nos tecidos [4]. No choque hemorrágico ocorre a hemodiluição, devido a perda de elementos celulares pela solução de continuidade dos vasos e também devido à hidremia [4, 30].

As diferenças observadas nos valores de hemoglobina no sangue, em relação ao método de abate utilizado (Tabela 1) pode ser devido a variações individuais como também ao método de abate propriamente dito. Todas as colheitas foram realizadas 5 segundos após o corte dos vasos. No abate realizado através de pistola pneumática seguido por estimulação elétrica apresentou menores valores de hemoglobina no sangue. A estimulação elétrica nesse processo era iniciada imediatamente após o corte dos vasos, portanto, a colheita ocorreu alguns segundos após o início de estimulação. Foi encontrada correlação positiva $(P<0,05)$ entre a idade dos animais e o teor de hemoglobina no sangue.

O processo de sangria pode ser avaliado pelo teor de hemoglobina residual na carne ou pela quantidade de sangue residual, medida pela relação entre o teor de hemoglobina na carne com o teor de hemoglobina sangüínea, o que denominamos eficiência da sangria [27].

A carne dos animais abatidos pelo método kasher apresentou valores de hemoglobina residual inferiores à carne obtida dos animais insensibilizados por pistola pneumática, sem apresentar diferenças em relação à carne dos animais insensibilizados através de marreta. A estimulação elétrica não afetou o teor de hemoglobina residual, confirmando as observações de GEROLD \& STOLE [11]. A hemoglobina residual apresentou alta correlação positiva $(P<0,01)$ com o peso da carcaça, contrariando os resultados apresentados por GEROLD \& STOLE [11]. A correlação positiva entre hemoglobina e mioglobina foi observada no nosso trabalho $(P<0,01)$, e na pesquisa de GEROLD \& STOLE [11]. 
A avaliação da eficiência da sangria (Tabela 1), demonstrou a presença de menor valor de sangue retido na carne dos animais abatidos pelo método kasher, em concordância com REGENSTEIN \& REGENSTEIN, e FOLHA DE SÃO PAULO, [8, 9, 24] sem diferença significativa para o método de abate com utilização de marreta e maior valor para os animais abatidos por pistola pneumática. A estimulação elétrica, efetuada após a sangria também apresentou efeito negativo na eficiência da sangria.

A avaliação de mioglobina e pigmentos totais ( $T a-$ bela 1) apresentou comportamento análogo em relação ao método de abate.

Animais mais pesados apresentaram menor eficiência de sangria, maior teor de hemoglobina residual, mioglobina e pigmentos totais (Tabela 2).

TABELA 1. Média dos valores obtidos na avaliação da hemoglobina sangüínea, hemoglobina na carne, eficiência da sangria, mioglobina e pigmentos totais.

\begin{tabular}{cccccc}
\hline $\begin{array}{c}\text { Método de } \\
\text { abate }\end{array}$ & $\begin{array}{c}\text { Hemoglobina } \\
\text { sangüinea }(\mathrm{g} / \mathrm{dL})\end{array}$ & $\begin{array}{c}\text { Hemoglobina na } \\
\text { carne }(\mathrm{g} / 100 \mathrm{~g})\end{array}$ & $\begin{array}{c}\text { Eficiência da } \\
\text { sangria }(\mathrm{g} / 100 \mathrm{~g})\end{array}$ & $\begin{array}{c}\text { Mioglobina } \\
(\mathrm{g} / 100 \mathrm{~g})\end{array}$ & $\begin{array}{c}\text { Pigmentos totais } \\
(\mathrm{g} / 100 \mathrm{~g})\end{array}$ \\
\hline Kasher & $16,4196 \mathrm{~b}^{*}$ & $0,3160 \mathrm{a}$ & $1,9809 \mathrm{a}$ & $0,3548 \mathrm{a}$ & $0,6591 \mathrm{a}$ \\
Marreta & $15,6768 \mathrm{~b}$ & $0,3281 \mathrm{a}$ & $2,1285 \mathrm{ab}$ & $0,3540 \mathrm{a}$ & $0,6575 \mathrm{a}$ \\
Pistola & $16,4722 \mathrm{~b}$ & $0,3830 \mathrm{~b}$ & $2,3226 \mathrm{~b}$ & $0,4217 \mathrm{~b}$ & $0,7804 \mathrm{~b}$ \\
pneumática & & & & & \\
$\quad$ Pistola & $14,7389 \mathrm{a}$ & $0,3802 \mathrm{~b}$ & $2,6069 \mathrm{c}$ & $0,3913 \mathrm{ab}$ & $0,7624 \mathrm{~b}$ \\
pneumática & & & & & \\
$\quad$ com & & & & & \\
estimulação & & & & & \\
elétrica & & & & & \\
\hline
\end{tabular}

Letras minúsculas diferentes, na mesma coluna, indicam haver diferença significativa $(P<0,05)$, pelo teste de Tukey.

TABELA 2. Correlações entre os parâmetros analisados $(n=240)$

\begin{tabular}{|c|c|c|c|c|c|c|}
\hline & Peso & $\begin{array}{l}\text { Hemoglobina } \\
\text { sanguinea }\end{array}$ & $\begin{array}{l}\text { Hemoglobina } \\
\text { na carne }\end{array}$ & $\begin{array}{l}\text { Eficiência } \\
\text { da sangria }\end{array}$ & Mioglobina & $\begin{array}{c}\text { Pigmentos } \\
\text { totais }\end{array}$ \\
\hline Idade & $0,2339 \mathrm{~ns}$ & $0,14515^{\star}$ & $-0,03728 \mathrm{~ns}$ & $-0,0836 \mathrm{~ns}$ & $0,16272^{*}$ & $0,09009 \mathrm{~ns}$ \\
\hline Peso & & $-0,09398 \mathrm{~ns}$ & $0,1783^{\star \star}$ & $0,20204^{\star \star}$ & $0,15423^{*}$ & $0,20094^{\star \star}$ \\
\hline $\begin{array}{l}\text { Hemoglobina } \\
\text { sangüinea }\end{array}$ & & & $0,05257 \mathrm{~ns}$ & $-0,45124^{\star *}$ & $0,15528^{\star}$ & $0,11101 \mathrm{~ns}$ \\
\hline $\begin{array}{l}\text { Hemoglobina } \\
\text { na carne }\end{array}$ & & & & $0,85513^{\star \star}$ & $0,57376^{\star *}$ & $0,85641^{\text {** }}$ \\
\hline $\begin{array}{l}\text { Eficiência da } \\
\text { sangria }\end{array}$ & & & & & $0,42996^{\star \star}$ & $0,70945^{\star \star}$ \\
\hline Mioglobina & & & & & & $0,89059^{\star \star}$ \\
\hline
\end{tabular}

ns = não significativo; ${ }^{*}=$ significativo $(P<0,05) ;{ }^{* *}=$ significativo $(P<0,01)$.

\section{4 - CONCLUSÕES}

O método de abate afeta sensivelmente o processo de sangria, sendo a eficiência maior no abate kasher e menor no abate realizado através da insensibilização por pistola pneumática, seguida imediatamente pela estimulação elétrica. A sangria é menos eficiente em animais de maior peso.

\section{5 - REFERÊNCIAS BIBLIOGRÁFICAS}

[1] ARGENTINA. MINISTÉRIO DA AGRICULTURA Y GANADERIA. Regulamento de inspeccion de productos, subproductos y derivados de origem animal: Decreto $4238 / 68$ y normas legales conexas. Buenos Aires, 1971.560p.
[2] BARBOSA da SILVA, C.A., coord. Matadouro misto de bovinos e suínos. Brasília: Ministério da Agricultura, do Abastecimento e da Reforma Agrária, Secretaria de Desenvolvimento Rural, 1995. 32p.

[3] BARTELS, H. Inspección veterinária de la carne. Zaragoza: Acribia, 1980. 491p.

[4] BOgOSSIAN, L. Choque. 3. ed. Rio de Janeiro: Atheneu, 1976. 443p.

[5] BRASIL. Ministério da Agricultura. Instrução Normativa $n^{\circ}$. 3 , de 07 de janeiro de 2000. Regulamento técnico de métodos de insensibilização para o abate humanitário de animais de açougue. S.D.A./M.A.A. Diário Oficial da União, Brasília, p.14-16, 24 de janeiro de 2000, Seção I. (Internet: www.agricultura.gov.br/das/dipoa/ Anexo\%20Abate.htm)

[6] DACIE, J.V., LEWIS, S.M. Practical haematology. London:Churchill Livingstone. 1975. 629p.

[7] DRABKIN, D.L., AUSTIN, J.H. Spectrophotometric studies. I. Spectrophotometric constants for common hemoglobin derivates in human, dog and rabbit blood. Journal of Biological Chemistry, Baltimore, v. 98, p. 719-733, 1932.

[8] FOLHA DE SÃO PAULO. O ritual do abate "Kasher". Folha de São Paulo, São Paulo, 28 mar. 1992, Cotidiano. p. 3-1.

[9] FOLHA DE SÃO PAULO. São Paulo ganha abatedouro 100\% Kasher. Folha de São Paulo, São Paulo, 01 set. 1992a, AgroFolha, p. 5-1.

[10] GANONG, W.F. Fisiologia médica. 4. ed. São Paulo: Atheneu, 1983. 665p.

[11] GEROLD, M., STOLLE, A. Elektrostimulation: untersuchungen über den einflub auf den ausblutungsgrad von rinderschalachttierkörpern. Fleischwirtschaft, Frankfurter, v. 74, n. 8, p. 864-869, 1994.

[12] GIL, J.I., DURÃO, J.C. Manual de inspeção sanitária de carnes. Lisboa: Fundação Caloustre Gulbenkian, 1985. 563p.

[13] GUYTON, A.C. Tratado de fisiologia médica. 4. ed. Rio de Janeiro: Guanabara Koogan, 1973. 975p.

[14] HEDRICK, H.B., ABERLE, E.D., FORREST, J.C., JUDGE, M.D., MERKEL, R.A. Principles of meat science. 3.ed., DUBUQUE:Kendal/Hunt Publ. Co., 1994, 354p.

[15] KARASZ, A.B., ANDERSEN, R., POLLMAN, R. Determination of added blood in ground beef. Journal of Association of Agriculture Chemistry, Champaing, v. 59, n. 6, p. 12401243, 1976.

[16] KOLB, E. ed. Fisiologia veterinária. 4 ed. Rio de Janeiro: Ed. Guanabara Koogan, 1984. 612p.

[17] LEACH, T.M. Pre-slaughter stunning. In: LAWRIE, R., ed. Developments in meat science - 3. London: Elsevier Appl. Sci. Publ., 1985. p. 51-87.

[18] MATOS, M.S., MATOS P.F. Laboratório clínico médico veterinário. Salvador: Arco-íris, 1981. 320p.

[19] MUCCIOLO, P. Carnes: estabelecimentos de matança e de industrialização. São Paulo:Íncone, 1985. 102p.

[20] PETTY, D.B., HATTINGH, J., GANHÃO, M.F., BEZEUIDENHOUT, L. Factors which affect blood variables of slughetered cattle. Tydskr. S. Afr. Veterinary Ver., v. 65, n. 2, p. 41-45, 1994

[21] PICCHI, V., AJZENTAL, A. Abate bovino segundo o ritual judáico. Revista Nacional da Carne, São Paulo, v. 18, n. 202, p. 53-57, 1993.

[22] PISKE, D. Aproveitamento de sangue de abate para alimentação humana. I. Uma revisão. Boletim do Instituto de Tecnologia de Alimentos, Campinas, v. 19. n. 3, p. 253308, 1982. 
[23] REGENSTEIN, J.M., REGENSTEIN, C.E. An introduction to the kosher dietary laws for food scientists and food processors. Food Tecnology, Chicago, v. 33, n. 1, p. 89-99, 1979.

[24] REGENSTEIN, J.M., REGENSTEIN, C.E. The kosher dietary laws and their implementation in the food industry. Food Tecnology, Chicago, v. 42, n. 6, p. 86-94, 1988.

[25] REGENSTEIN, J.M., REGENSTEIN, C.E. Current issues in kosher foods. Trends in Food Science Tecnology, n. 3, p. 50-54, 1991.

[26] ROÇA, R.O. Influência do banho de aspersão "ante-mortem em parâmetros bioquímicos e microbianos da carne bovina. Campinas, 1993. 185p. Tese (Doutorado em Engenharia de Alimentos, Área de Tecnologia de Alimentos) Faculdade de Engenharia de Alimentos - Universidade Estadual de Campinas UNICAMP.

[27] ROÇA, R.O., SERRANO, A.M., Influência do banho de aspersão ante-mortem em parâmetros bioquímicos e na eficiência da sangria da carne bovina. Pesquisa Agropecuária Brasileira, Brasília, v. 30, n. 8, p. 1107-1115, 1995.

[28] SANZ EGAÑA. Enciclopedia de la carne. Madrid: Espasacalpe, 1967. 1095p.

[29] SAS User's procedures guide. Version 6. 4. ed. Vol.1-2, Cary, NC:SAS Institute, Inc, 1989. 1686p.

[30] SCHALM, O.W., JAIN, N.C., CARROLL, E.J. Veterinary hematology. Philadelphia: Lea, Febiger. 1975. 807p.

[31] SNEDECOR, G.W., COCHRAN, W.G. Statistical methods. 6 ed. Ames: lowa State University Press, 1978. 593p.
[32] SWATLAND, H.J. Slaughtering. Internet: http:// www.bert.aps.uoguelph.ca/ swatland/ch1.9.htm. 1999. 10p.

[33] SMULDERS, F.J.M., EIKELENBOOM, G., LAMBOOY, E., et al. Electrical stimulation during exsanguination, effects on the prevalence of blood splash and sensory quality characteristics in veal. Meat Science, Oxon, v. 26, n. 2, p. 89-99, 1989.

[34] THORNTON, H. Compêndio de inspeção de carnes. Londres: Bailliere Tindall an Cassel, 1969, 665p.

[35] VIMINI, R.J., FIELD, R.A., RILEY, M.L., et al Influence of delayed blending after stunning on beef muscle characteristics. Journal of Animal Science, Champaing, v. 56, n. 3, p. 608-615, 1983.

[36] VIMINI, R.J., FIELD, R.A., RILEY, M.L., et al Effect of delayed bleending after captive bolt stunning on heart activity and blood removal in beef cattle. Journal of Animal Science, Champaing, v. 57, n. 3, p. 628-631, 1983a.

[37] WARRISS, P.D. The residual blood content of meat. A review. Journal of Science Food Agriculture, London, v. 28, p. 457-462, 1977.

\section{AGRADECIMENTOS}

À FAPESP pelo auxílio financeiro, ao CNPq, pela bolsa de Produtividade em Pesquisa concedida, ao Serviço de Inspeção Federal - POINS Bauru, e aos Frigoríficos Mondelli, de Bauru e Bertin, de Lins. 